\title{
Managing the baby with a patent ductus arteriosus. More questions than answers?
}

\section{P W Fowlie}

\section{There is no evidence that treatment of patent ductus arteriosus results in long term benefit}

P remature infants with a patent ductus arteriosus (PDA) are at increased risk of more prolonged and more severe respiratory distress syndrome, bronchopulmonary dysplasia, and death than similar infants whose ductuses have closed. In an attempt to improve the outcome for these infants, three broad strategies have been proposed: prophylactic "closure", closure of the asymptomatic but clinically detected PDA, and closure of the symptomatic PDA. Interventions, some of which have been subjected to more rigorous scrutiny than others, used as part of these strategies include fluid restriction, the use of diuretics, the use of cyclo-oxygenase inhibitors, and surgical closure using a variety of surgical techniques. There are now seven completed systematic reviews in the Cochrane library looking at such interventions. ${ }^{1}$ The bulk of the evidence available assesses the impact on short term outcomes, with some of the strategies resulting in, for example, an apparent decrease in the risk of developing a symptomatic PDA or a reduction in duration of oxygen dependence. These short term benefits have to be balanced against adverse effects such as temporary renal impairment and a possible increase in the risk of necrotising enterocolitis. Importantly, there is no evidence available that any of the strategies examined result in long term benefit, in particular, disability-free survival, and for some strategies-for example, prophylactic intravenous indometacin-clear evidence exists that there is no long term benefit. Much uncertainty about "best practice" therefore remains. ${ }^{2}$

In this month's journal, Brooks et al report an observational study that explores the outcome for preterm infants born at the turn of the century whose ductuses failed to close. ${ }^{3}$ Using statistical modelling and regression analyses to correct for potential confounding variables, they suggest that infants with a persistent PDA are at increased risk of dying compared with those infants in whom the ductus closes spontaneously or in whom it is closed medically (OR $4.02 \quad$ (95\% CI 1.12 to 14.51)). This seemingly dramatic result might naturally lead clinicians to wonder whether or not closing the ductus surgically in these babies would improve survival. It should be noted, however, that none of the strategies tested in randomised clinical trials to date that effectively close the duct seem to result in improved survival, and such a hypothesis would need to be tested more rigorously.

Brooks et al suggest that a robust assessment of this issue might best be achieved by conducting a randomised controlled trial presumably comparing surgical closure with non-closure in infants whose ductuses remain patent in the neonatal period despite attempt(s) to close them medically. The design of such a study would need very careful consideration, however. Cardiac services are now highly centralised in most western countries, and very few infants will be in a situation where they can have their patent ductus ligated surgically on the neonatal unit. In most cases, any consideration of surgery is likely to depend on transporting the neonate at the very least a few hundred yards down a corridor and more likely many miles, sometimes hundreds of miles, to the nearest cardiac centre. Although in some parts of the world neonatal transport services are highly developed, in others they remain ad hoc, and the quality of transport care along with its impact on neonatal outcome is relatively unstudied. What is clear, however, is that this aspect of any infant's care-the transfer-would have to be considered an integral part of the intervention being tested. This would be analogous to the seminal UK Collaborative ECMO Trial $^{4}$ where a coordinated retrieval service was established for the trial, and babies were randomised to either travel to an extracorporeal membrane oxygenation (ECMO) centre or to "stay put". In addition, long term neurodevelopmental follow up was an integral feature of the trial. ${ }^{5}$ The whole episode of care, whether or not the infant ended up receiving ECMO or not, was then reported including an intention to treat analysis ${ }^{6}$ providing a true picture of the impact such a service might have on both short and long term infant outcomes. A similar study design may be appropriate to test this current hypothesis.

In the meantime, neonatologists will have to consider the options available. Are the short term gains in actively treating PDA worth the potential adverse effects when there is scant evidence of longer term benefit? And in relation to surgical closure when the duct remains open, particularly when this might involve interhospital transfer of an unwell baby, what might be the impact of the transfer itself? Certainly much more evidence is needed before this can be recommended as "best practice".

Arch Dis Child Fetal Neonatal Ed 2005;90:F190.

doi: $10.1136 /$ adc. 2004.065946

Correspondence to: Dr Fowlie, Neonatal Intensive Care Unit, Ninewells Hospital and Medical School, Dundee DDI 9SY, Scotland, UK; peter.w.fowlie@tuht.scot.nhs.uk

Competing interests: PWF has conducted and published a systematic review on the use of prophylactic indometacin in preterm infants.

\section{REFERENCES}

1 http://www.thecochranelibrary.com.

2 Wyllie J. Treatment of patent ductus arteriosus. Semin Neonatol 2003;8:425-32.

3 Brooks JM, Travadi JN, Patole SK, et al. Is sirgical ligation of a patent ductus arteriosus necessary? The Western Australian experience of conservative management. Arch Dis Child Fetal Neonatal Ed 2005;90:F235-9.

4 UK Collaborative ECMO Trail Group. UK collaborative randomised trial of neonatal extracorporeal membrane oxygenation. Lancet 1996:348:75-82.

5 Bennett CC, Johnson A, Field DJ, Elbourne D. UK Collaborative ECMO Trial Group. UK collaborative randomised trial of neonata extracorporeal membrane oxygenation: follow-up to age 4 years. Lancet 2001;357:1094-6.

6 Fergusson D, Aaron SD, Guyatt G, et al. Postrandomisation exclusions: the intention to treat principle and excluding patients from analysis. BMJ 2002;325:652-4. 
Neonatal research

\section{Participation in multiple neonatal research studies}

\section{Ward Platt}

\section{Doctors and members of ethics committees need guidance that is} underpinned by sound evidence on parental attitudes to research

I: $\mathrm{n}$ recent years, the journal has carried several papers on parental consent (assent) for participation of their babies in clinical trials. ${ }^{12}$ This is important because Archives is read by many of those who organise neonatal research projects. The approach to parents is often fraught with ethical dilemmas.

Clinicians and members of ethics committees alike need guidance that is underpinned by sound research evidence on parental attitudes and beliefs about participating in research, rather than well meant assumptions about how parents might feel. Readers of the Journal of Medical Ethics would find little of direct use when confronted by the clinical realities and research questions of neonatal care-although this journal once published a neonatal study on the variability of ethics committee views. Rather, it is to specialist or general clinical journals that clinicians look for good information in relation to parental views and perceptions, and for an evidence base on the process of consent.

Mostly, existing work has concentrated on consent for inclusion in single trials. Yet in many large tertiary centres, there is the likelihood that several studies are in progress simultaneously. Some may be local, but others may involve participation in large multicentre studies. Either way, it is likely that the experience of parents will be different if approached serially for a number of studies; and some ethics committees may take views on whether more than one approach is ethical. So it follows that we need good evidence in relation to parents participating in one or more trials.
The two papers on this topic that we carry this month ${ }^{3}$ reinforce each other, and give us confidence that in general it will not be inappropriate to approach parents more than once in relation to clinical trials. The paper from Melbourne centred on a tertiary neonatal intensive care unit, and that from Philadelphia focused on neonates undergoing cardiac surgery. The first was dominated by babies at high risk because of prematurity, and was specifically concerned with the question of recruitment to multiple studies, whereas the second concerned larger babies at risk from major cardiac surgery, and, although primarily about parental attitudes in general, it involved parents whose babies could be recruited to a number of different studies.

What do we learn from these papers? As with research on consent for single studies, altruism was a strong driver, coupled with a general perception of the benefit of both research and participation in research; perhaps also there was consciousness that an environment in which research was undertaken was likely to be at the cutting edge of the specialty. A possible but tantalisingly elusive implication of the Philadelphia data is that rates of participation may have related to the nature of the study in question. This surely requires further investigation. Most importantly, engagement in more than one study did not emerge as a major problem. Many parents declined participation in some studies, although only $10 \%$ declined routinely, but that does not mean that they should not have been asked.
Indeed, given the flavour of the reasons for participation, would it be fair to deny parents the possibility of enrolling their baby in extra studies if their babies were eligible? And if parents decline participation in a study early in their baby's life, should we extrapolate that refusal to other studies at later times? Probably not-but here again, more research would be very useful.

Then there is what we don't learn. There is little research in general, and nothing in these studies, on the beliefs and attitudes of ethnic minorities, in either the United Kingdom or other countries into which there has been significant migration. What we cannot do is extrapolate from information obtained in western European or American culture to people of other cultures. This too would be fertile ground for future research.

We should never forget the fact that parents talk to each other, and many of us will have had the experience of parents approaching us about possible participation in a study even before we have raised the issue with them. The next challenge will be to ensure that the members of research ethics committees and review boards become aware of this information. But that's a separate problem.

Arch Dis Child Fetal Neonatal Ed 2005; 90:F191.

doi: 10.1136/adc.2004.067371

Correspondence to: Dr M P Ward Platt, Ward 35, Royal Victoria Infirmary, Newcastle upon Tyne NE1 4LP, UK; m.p.ward-platt@nd.ac.uk

Competing interests: none declared

\section{REFERENCES}

1 Stenson BJ, Becher JC, Mclntosh N. Neonatal research: the parental perspective. Arch Dis Child Fetal Neonatal Ed 2004;89:F321-3.

2 Burgess E, Singhal N, Amin H, et al. Consent for clinical research in the neonatal intensive care unit: a retrospective survey and a prospective study. Arch Dis Child Fetal Neonatal Ed 2003;88:F280-5.

3 Morley CJ, Lau R, Davis PG, et al. What do parents think about enrolling their premature babies in several research studies? Arch Dis Child Fetal Neonatal Ed 2005;90:F225-8.

4 Hoehn KS, Wernovsky G, Rychik J, et al. What factors are important to parents making decisions about neonatal research? Arch Dis Child Fetal Neonatal Ed 2005;90:F267-9. 


\section{Standardised feeding regimens: hope for reducing the risk of necrotising enterocolitis}

\section{S S Premii}

\section{A perspective on the paper by Patole and de Klerk'}

$\mathrm{N}$ ecrotising enterocolitis (NEC), an acquired gastrointestinal disease in neonatal intensive care unit survivors, affects one to three infants per 1000 live births and is associated with significant mortality and morbidity. ${ }^{2}$ Although it has not been proven, many believe that, in premature infants, a precursor to NEC is feeding intolerance, specifically, prefeed gastric residuals or bile stained aspirates. ${ }^{4-6}$ These associated intestinal signs of NEC may also reflect a delay in maturation of the neonate's motor activity such that they lack complete interdigestive cycles during fasting. As no biological markers exist to diagnose NEC, clinical wisdom guides decision making related to its diagnoses and management. Furthermore, there is a paucity of research identifying feeding practices, except for breast milk feeds, that offer the greatest potential benefit against developing NEC. Moreover, hormonal, anatomical, and functional limitations of low birthweight infants, the additive effects of critical illness, and intrauterine environmental factors-for example, antenatal glucocorticoids-complicate feeding decisions in this population of infants. Consequently, there is great variability in feeding orders for low birthweight infants.

A standardised feeding regimen (SFR) is one strategy to address the challenges of feeding low birthweight infants. Establishing such an SFR would require synthesising the available evidence $^{7}$ and communicating the clinical wisdom from the experts, thereby promoting a more systematic approach to feeding low birthweight infants. A systematic review and meta-analysis of observational studies reporting the incidence of NEC "before" and "after" implementation of SFR undertaken by Patole and de Klerk ${ }^{1}$ reported a pooled risk ratio of $0.13 \quad(95 \%$ confidence interval 0.03 to 0.50$)$. The reduced incidence of NEC after the introduction of SFRs was attributed to minimisation of variations in enteral feeding practices. $^{1}$ However, it is unclear what degree of variation in practice will significantly contribute to the incidence of NEC. Alternatively, what measure of reduction in variation in practice resulting from the implementation of SFRs will significantly contribute to a decrease in the incidence of NEC?

In order for an SFR to attain the goal of preventing or minimising NEC, compliance or adherence to the regimen is imperative. The issue of compliance with the SFR may also contribute to the significant heterogeneity $(\mathrm{p}<0.001)$ noted between studies included in the systematic review by Patole and de Klerk. ${ }^{1}$ One must consider ethical conflict when addressing the issue of noncompliance. Uncertainty is reduced in SFRs through the prudent use of evidence from rigorous trials or, in the absence of such trials, expert clinical opinion. The intent is to minimise the art of medicine and promote the use of clinical science. ${ }^{8}$ The validity of the SFR will be limited if the evidence on which it is based is weak. ' In addition, SFRs are not intended to be prescriptive, and their use in clinical practice will ultimately require the practitioner's judgment. The consensus of experts, which has been the crux of the development of SFRs, may eliminate idiosyncratic judgments of single clinicians; however, it introduces value judgmentsfor example, choice made on the basis of safety or effectiveness-about what ought to be done for a condition. ${ }^{10}$ Understanding these ethical conflicts or dilemmas will help to facilitate implementation or revision of guidelines and increase the potential benefit to low birthweight infants. Future studies evaluating SFRs need to have a qualitative component that identifies and defines ethical issues, explains ethical judgments or behaviours, and analyses or appraises clinical decision making.

Patole and de Klerk ${ }^{1}$ propose that clinical variation in practice determines risk of NEC. Although not explicitly stated, one can infer that this refers to variability in physician orders. However, nursing management of tube feeding is also inconsistent and varies from nurse to nurse. ${ }^{11}$ Each nurse uses their individual expertise to resolve problems related to regurgitation, abdominal distension, and residuals. ${ }^{12}$ Hodges and Vincent $^{13}$ have shown variability in the practice of withdrawing feeding and management of feeding residuals. As the success of any nutritional approach depends on neonatal nurses, who spend a significant amount of time feeding, and assessing infants before, during, and after feeds, ${ }^{11}$ a better understanding of nursing practice related to tube feeding is required. This understanding will facilitate a unified systematic approach to nursing management of tube feeds based on the current state of scientific knowledge.

Patole and de Klerk $^{1}$ attribute the reduced incidence of NEC after the introduction of an SFR to an increased awareness leading to early detection and management of signs of "feeding intolerance". However, there is no consensus in the literature with regard to the operational definition of feeding intolerance. In the scientific literature, prefeed gastric residual volumes, colour of gastric aspirates, abdominal distension, spitting up, presence of blood in stool, and apnoea and bradycardia are signs listed for feeding intolerance. ${ }^{614-16}$ Researchers have tried to define feeding intolerance by quantifying volume of prefeeding gastric residuals considered to be significant; however, the volume considered significant varies across studies. The threshold of what is considered to be a significant volume of gastric residual appears to be increasing. ${ }^{17}$ The chances that clinicians will adopt SFRs decreases with an increase in uncertainty of the validity of the science behind them. A standardised approach to identifying feeding intolerance and predicting NEC will facilitate compliance with SFRs, as well as facilitate a more meaningful interpretation of studies that examine the relation between diet and gastrointestinal diseases such as NEC. One potential strategy is the development of decision rules that have been assessed for reliability and validity.

The process by which SFRs are shared and eventually adopted is a social process which influences the clinician's knowledge, attitudes, and behaviour. ${ }^{18} 19$ Consequently it is not surprising that Patole and de Klerk ${ }^{1}$ note that it is the process rather than the specific constituents that lead to improved outcomes. A Cochrane systematic review on guidelines in professions allied to medicinefor example, nursing, midwifery, and health visiting_identified 18 studies that provide evidence that guideline driven care can be effective in changing the process of care. ${ }^{20}$ 
In conclusion, an SFR offers hope for reducing the risk of NEC by decreasing variability in practice. SFRs should address variability in both medical and nursing practice. Implementation strategies that comprise processes aimed to improve the clinician's compliance with the recommendations will determine the extent to which they are useful. It is imperative, however, that clinicians understand the values driving research, outcomes, and management issues. If clinicians lack this understanding, then ethical conflict or dilemmas could ensue which may impede the adoption of the SFR. In addition, SFRs may not be appropriate for all low birthweight infants, hence, clinicians need to exercise judgment otherwise they may compromise the infant's care. Future studies need to measure the relative effectiveness of the SFR. Emphasis on effectiveness will allow the researcher to evaluate the utility of the SFR in practice, process of care, quality of care, and patient/parent satisfaction. ${ }^{21}$

Arch Dis Child Fetal Neonatal Ed 2005;90:F192-F193.

doi: 10.1136/adc.2004.063198

Correspondence to: Dr Premii, University of Calgary, Faculty of Nursing, 2500 University Dr NW, Calgary, AB, Canada, T2N 1N4; premjis@ucalgary.ca
Competing interests: none declared

\section{REFERENCES}

1 Patole S, de Klerk N. Impact of standardised feeding regimens on incidence of neonatal necrotising enterocolitis: a systematic review and meta analysis of observational studies. Arch Dis Child Fetal Neonatal Ed 2005;90:F147-51.

2 Guthrie S, Gordon P, Thomas V, et al. Necrotizing enterocolitis among neonates in the United States. J Perinatol 2003;23:278-85.

3 Lee J, Polin R. Treatment and prevention of necrotizing enterocolitis. Semin Neonatal 2003;8:449-59.

4 Bell M, Temberg J, Feigin R, et al. Neonatal necrotizing enterocolitis. Therapeutic decisions based upon clinical staging. Ann Surg 1978;187:1-7.

5 Walsh M, Kliegman R. Necrotizing enterocolitis: treatment based on staging criteria. Pediatr Clin North Am 1986;33:179-201.

6 Mihatsch W, von Schoenaich P, Fahnenstich H, et al. The significance of gastric residuals in the early enteral feeding adancement of extremely low birth weight infants. Pediatrics 2002; 109:457-9.

7 Lewis S. Paradox, process and perception: The role of organizations in clinical practice guidelines development. CMAJ 1995; 153:1073-7.

8 Jonsen A, Siegler M, Winslade W. Clinical ethics: a practical approach to ethical decisions in clinical medicine, 5th ed. Toronto: McGraw-Hill, 2002

9 Hayward R, Wilson M, Tunis S, et al. Users' guides to the medical literature. VIII. How to use clinical practice guidelines. A. Are the recommendations valid? JAMA 1995;274:570-4.
10 Battista R, Hodge M, Vineis P. Medicine, practice and guidelines: the uneasy juncture of science and art. J Clin Epidemiol 1995:48:875-80.

11 Premii S, Paes B, Jacobson K, et al. Evidencebased feeding guidelines for very low-birthweight infants. Adv Neonat Care 2002;2:5-18.

12 Bragdon D. A basis for the nursing management of feeding the premature infant. J Obstet Gynecol Neonatal Nurs 1983;12:51S-7S.

13 Hodges C, Vincent P. Why do NICU nurses not refeed gastric residuals prior to feeding by gavage? Neonat Netw 1993:12:37-40.

14 Rayyis S, Ambalavanan N, Wright L, et al. Randomized trial of "slow" versus "fast" feed advances on the incidence of necrotizing enterocolitis in very low birth weight infants J Pediatr 1999;134:293-7.

15 Dollberg S, Kuint J, Mazkereth R, et al. Feeding tolerance in preterm infants: randomized trial of bolus and continuous feeding. J Am Coll Nutr 2000; 19:797-800.

16 Akintorin S, Kamat M, Pildes R, et al. A prospective randomized trial of feeding methods in very low birth weight infants. Pediatrics 1997:100:e4.

17 Premii S, Chessel L, Paes B, et al. A matched cohort study of feeding practice guidelines for infants weighing less than $1,500 \mathrm{~g}$. Adv Neonat Care 2002;2:27-36.

18 Cabana M, Rand C, Powe N, et al. Why don't physicians follow clinical practice guidelines? JAMA 1999;282:1458-65.

19 Mittman B, Tonesk X, Jacobson P. Implementing clinical practice guidelines: social influence strategies and practitioner behavior change. $Q R B$ Qual Rev Bull 1992;18:413-22.

20 Thomas L, Cullum N, McColl E, et al. Guidelines in professions allied to medicine. Cochrane Library. Issue 3. Oxford: Update Software, 2004.

21 Johanson J. Outcomes research, practice guidelines, and disease management in clinical gastroenterology. J Clin Gastroenterol 1998;27:306-11.

\section{Prevalence, causes, and outcome at 2 years of age of newborn encephalopathy}

\section{N Marlow, H Budge}

\section{A commentary on the article by Pierrat et al}

$\mathrm{R}$ gional population based studies of infants who suffer from intrapartum hypoxia are rare, and Pierrat and colleagues are to be congratulated on such a study. As always it is easy to criticise such studies because case definition is so difficult, and, without accurate imaging and detailed case evaluation, it is difficult to be sure that a neonatal encephalopathy is due to hypoxia. The definition of perinatal hypoxia-ischaemia that they have used might be viewed as inclusive and is at variance with the template for intrapartum causation for cerebral palsy, which requires evidence of an intrapartum event. ${ }^{1}$ Without detailed evaluation of each case, it is difficult to be certain of the timing of the cause.

In the literature, most outcome evaluations of neonatal populations have studied very preterm infants, and there have been only a few population studies of neonatal encephalopathy. The birth prevalence of encephalopathy reported in this paper is in keeping with the results of the Trent Neonatal survey (Department of Health Sciences, University of Leicester, Leicester LE 1 6TP, UK), which has prospectively collected well validated information for over 10 years. This study includes all children with seizures as a pragmatic definition of encephalopathy and reports population rates in the Trent Region of the United Kingdom varying from 1.3 to 1.4 per 1000 live births between 1999 and 2003. Neither study approaches the reported prevalence from Western Australia, ${ }^{2}$ but the latter was also a deliberately inclusive study. All three studies use different definitions.

In trying to understand the prevalence and outcome of intrapartum hypoxia, this study shows the need for accurate and clear case definition and for the role of obstetric factors, routine collection of cord blood gas data, and neonatal imaging with magnetic resonance imaging in teasing out the cause. All neonatal services should collect this information. The best definition of encephalopathy remains the three categories described first by Sarnat and Sarnat ${ }^{3}$ with or without the presence of seizures. A consensus over definition of encephalopathy is perhaps required in situations where detailed neurological assessment has not been carried out and for epidemiological purposes, although 
perhaps better would be to define a more useful perinatal dataset to allow better population data collection.

Cerebral palsy is perhaps the most important outcome in a study such as this. The prevalence of other disability such as visual impairment, deafness, and cognitive impairments is almost as important and would provide additional information on the level of disability, which is often severe after damaging intrapartum hypoxia. Pragmatic and accurate data collection is preferable to more detailed information without accuracy or universal coverage. We have standards to which our outcome studies for preterm children should aspire ${ }^{4}$ and definition of health status ${ }^{5}$ that has proved to be reliable in this group. ${ }^{6}$ It may now be time for some consensus over case definition, follow up, and outcome definition for the encephalopathic newborn.

\section{Arch Dis Child Fetal Neonatal Ed 2005;90:F193-F194.}

doi: 10.1136/adc.2004.057059

\section{Authors' affiliations}

N Marlow, H Budge, Academic Division of Child Health, School of Human Development, University of Nottingham, Nottingham, UK

Correspondence to: Professor Marlow, Academic Division of Child Health, Level E East Block, Queen's Medical Centre, Nottingham NG7 2UH, UK; neil.marlow@nottingham.ac.uk Competing interests: none declared

\section{REFERENCES}

1 MacLennan A. A template for defining a causal relation between acute intrapartum events and cerebral palsy: international consensus statement. BMJ 1999;319:1054-9.

2 Badawi N, Kurinczuk Jj, Keogh JM, et al. Antepartum risk factors for newborn encephalopathy: the Western Australian casecontrol study. BMJ 1998;317:1549-53

3 Sarnat HB, Sarnat MS. Neonatal encephalopathy following fetal distress. A clinical and electroencephalographic study. Arch Neurol 1976;33:696-705.

4 Mutch LM, Johnson MA, Morley R. Follow up studies: design, organisation, and analysis. Arch Dis Child 1989;64:1394-402.

5 Report of two working groups. Disability and perinatal care. Oxford: NPEU \& Oxford HA 1995

6 Jones HP, Guildea ZE, Stewart JH, et al. The Health Status Questionnaire: achieving concordance with published disability criteria. Arch Dis Child 2002;86:15-20

\section{Working in paediatrics and not a member of the Royal College of Paediatrics} and Child Health?

Have you thought about the benefits of joining?

Benefits and entitlements of membership

- Regular mailings including quarterly newsletter

- Listed in handbook plus your own copy at no extra charge

- Representation on Council (the College's governing body)

- Local support

- Archives of Disease in Childhood (BMJ cost to non members £206.00)

- Discount at Spring meeting

- Book club with discounts on books related to paediatrics

- CPD monitoring (fellows and associates)

- Voting at general meetings (fellows and ordinary members)

- Membership certificate (fellows and ordinary members)

- HST monitoring (ordinary members)

- Designation MRCPCH (ordinary members)

- Designation FRCPCH (fellows)

$\begin{array}{ll}\text { Subscriptions } & \text { (inclusive of Archives) } \\ \text { Junior } & £ 140.00 \\ \text { Ordinary } & £ 325.00 \\ \text { Fellow } & £ 380.00 \\ & \\ \text { Associate } & £ 238.00\end{array}$

Concessionary subscriptions are available for those working part time or resident overseas.

Application forms and information on eligibility are available via www.rcpch.ac.uk or by contacting The Membership Section, Royal College of Paediatrics and Child Health, 50 Hallam Street, London WIW 6DE; tel (020) 7307 5619/20/23; fax (020) 7307 5601; email: membership@rcpch.ac.uk. 